TRANSACTIONS OF THE

AMERICAN MATHEMATICAL SOCIETY

Volume 354, Number 6, Pages 2327-2339

S 0002-9947(02)02983-5

Article electronically published on February 12, 2002

\title{
ISOPERIMETRIC REGIONS IN CONES
}

\author{
FRANK MORGAN AND MANUEL RITORÉ
}

\begin{abstract}
We consider cones $C=0 ※ M^{n}$ and prove that if the Ricci curvature of $C$ is nonnegative, then geodesic balls about the vertex minimize perimeter for given volume. If strict inequality holds, then they are the only stable regions.
\end{abstract}

\section{INTRODUCTION}

An isoperimetric region minimizes perimeter for given volume. There are few manifolds of dimension $n+1 \geqslant 3$ for which the isoperimetric regions are known: the classical examples $\mathbb{R}^{n+1}, \mathbb{S}^{n+1}, \mathbb{H}^{n+1}$, certain Cartesian products $[\mathrm{P}],[\mathrm{PR}]$, $\left[\mathrm{R} 1\right.$, and $\mathbb{R P}^{3}[\mathrm{RR}$. To this list we add cones with nonnegative Ricci curvature by showing that geodesic balls about the vertex are isoperimetric (Thm. 3.6 Cor. 3.9. If $\mathrm{Ric}>0$, then these are the only smooth regions with nonnegative second variation of perimeter (for fixed volume).

The proof. The proof shows first that isoperimetric regions exist. By standard geometric measure theory, their boundaries are smooth constant-mean-curvature hypersurfaces except possibly for the vertex and a singular set of Hausdorff dimension at most $n-7$. Following earlier work of Barbosa and do Carmo $[\mathrm{BdC}]$ and Montiel [Mo], we use the Minkowski formulas (Prop. 3.4) and a variation vectorfield associated to homotheties of the cone to show that the only stable regions are the geodesic balls about the vertex (or flat round balls with greater perimeter). To generalize the second variation argument to possibly singular hypersurfaces in cones, we show that the singular sets are neglibible, using a new covering argument (Lemma 3.1.

Existence. Geometric measure theory provides us with the existence of a limiting, perimeter-minimizing region, but some volume may disappear to infinity. As long as the limit is not 0 , a rescaling has the desired volume. If on the other hand everything disappears to infinity, an isoperimetric inequality (Thm. 2.1) after Bérard and Meyer $[\mathrm{BM}]$ shows that a geodesic ball about the vertex does better.

Acknowledgements. This work was carried out principally while Morgan was visiting the University of Granada in the spring of 1999. Morgan's work is partially supported by a National Science Foundation grant. Ritoré's work is partially supported by DGICYT research group PB97-0785.

Received by the editors May 23, 2001 and, in revised form, November 1, 2001.

2000 Mathematics Subject Classification. Primary 53C42; Secondary 49Q20.

(C) 2002 by the authors 
We begin with an easy theorem about spherically symmetric cones by comparison with Euclidean space, as in Bray ( $\mathrm{Br} \S 2.5]$, see [BrM, Cors. 2.3, 2.6]).

Theorem 1.1 (Isoperimetric Regions in Spherical Cones). Let $C$ be the cone over a non-great round sphere $S^{n}$ in $\mathbb{S}^{N} \subset \mathbb{R}^{N+1}(n \geqslant 1)$. Then in $C$, for given volume, a round sphere $T_{0}$ about the vertex uniquely minimizes perimeter (among smooth surfaces or more generally among integral currents).

Proof. Consider the natural map $f: C \rightarrow \mathbb{R}^{n+1}$ (mapping $S$ homothetically to a great sphere), stretching by a factor $\lambda>1$ in tangential directions. The map $f$ multiplies all volume by $\lambda^{n}$, tangential area by $\lambda^{n}$, and other area by a factor less than $\lambda^{n}$. The sphere $T_{0}$ in $C$ is stretched by the maximum possible factor $\lambda^{n}$. Since its image $f\left(T_{0}\right)$, a round sphere in $\mathbb{R}^{n}$, is minimizing, $T_{0}$ must be minimizing. Any minimizer has to be a completely tangential inverse image of a round sphere; $T_{0}$ is therefore the only minimizer.

\section{EXISTENCE AND REgUlarity}

Theorem 2.1 (Isoperimetric Inequality (Bérard-Meyer [BM, Appendix C])). Let $M^{n+1}$ be a smooth, complete Riemannian manifold, possibly with boundary, of bounded geometry (bounded sectional curvature and positive injectivity radius). Then, given $0<\delta<1$, there exists $V_{0}>0$ such that any open set $U$ of volume $V \leqslant V_{0}$ satisfies

$$
|\partial U| \geqslant \delta \beta V^{n /(n+1)},
$$

where

$$
\left|\mathbb{S}^{n}\right|=\beta\left|\mathbb{B}^{n+1}\right|^{n /(n+1)} \text { in } \mathbb{R}^{n+1} .
$$

Here $|\partial U|$ denotes the $n$-dimensional Hausdorff measure of the topological boundary of $U$ (or, better, the mass of the current boundary).

Proof. The result differs from the treatment in [BM] in two minor respects. First of all, Bérard and Meyer consider only smooth regions, but any open set of finite perimeter may be approximated by a smooth, bounded region of nearly the same volume and perimeter.

Second, Bérard and Meyer consider only compact manifolds $M$ for their global result (although they remark that their local result applies to complete manifolds with boundary). Given small $\rho>0$, they cover $M$ with $\ell$ small balls $B\left(x_{i}, \rho\right)$, such that the $B\left(x_{i}, \rho / 2\right)$ are disjoint. We may need to use a covering by countably many such balls, constructed for example over an increasing exhaustive sequence of compact subsets. Next, for each $i$ they choose $\rho<t_{i}<2 \rho$ such that

$$
\left|\partial B\left(x_{i}, t_{i}\right) \cap U\right| \leqslant \frac{V}{\rho},
$$

and conclude that

$$
\sum_{i=1}^{\ell}\left|\partial B\left(x_{i}, t_{i}\right) \cap U\right| \leqslant \ell \frac{V}{\rho} .
$$

We note that actually

$$
\sum_{i}\left|\partial B\left(x_{i}, t_{i}\right) \cap U\right| \leqslant \frac{\left|B\left(x_{i}, 2 \rho\right) \cap U\right|}{\rho} .
$$


Moreover, since the sectional curvature is bounded, volume estimates show that the number of $B\left(x_{i}, 2 \rho\right)$ containing any point is bounded by some constant $\ell^{\prime}$. Hence we can conclude that

$$
\sum_{i}\left|\partial B\left(x_{i}, t_{i}\right) \cap U\right| \leqslant \frac{\ell^{\prime} V}{\rho} .
$$

The rest of the argument is unchanged. Namely, in each (small) component of $M-\bigcup \partial B\left(x_{i}, t_{i}\right)$, which is closely approximated by its Euclidean tangent space, they can apply a local isoperimetric inequality. By (2.2), the edge effects are bounded by a constant times $V$, which for small $V$ is much less than perimeter.

Theorem 2.2 (Existence and Regularity Theorem). Let $C^{n+1}=0 \nVdash M^{n} \subset \mathbb{R}^{N+1}$ $(n \geqslant 1)$ be the cone over a smooth submanifold $M^{n}$ of the sphere $\mathbb{S}^{N}$, with $\left|M^{n}\right|<$ $\left|\mathbb{S}^{n}\right|$. Then for all $V>0$, there exists a bounded open set $U \subset C$ of volume $V$ minimizing the Hausdorff measure of $\partial U$. Moreover, $\partial U$ is a smooth, constantmean-curvature submanifold, except possibly for 0 and a singular set of Hausdorff dimension at most $n-7$.

Proof. It suffices to prove the result in the category of locally integral currents, since the Hausdorff measure of the topological boundary of an open set of finite volume is greater than or equal to the mass of its current boundary ( $\mathrm{F}, 4.5 .12$, 4.5.6]). Consider a sequence $U_{i}$ of locally integral currents of mass $V$ and boundary mass approaching the infimum $m_{0}$. By compactness ([M1, 9.1], [S, 27.3, 31.2]) we may assume that the sequence converges to a locally integral current $U$. By standard arguments, $U$ is perimeter-minimizing for its volume $V^{\prime} \leqslant V$. If $U \neq 0$ (and hence $V^{\prime}>0$ ), a rescaling under homothetic expansion of $C$ yields a minimizer with volume $V$ as desired.

Alternatively, suppose $U=0$. Choose $0<\delta<1$ such that $\left(|M| /\left|\mathbb{S}^{n}\right|\right)^{1 /(n+1)}<$ $\delta^{2}$. Choose $V_{0}$ to obtain the isoperimetric inequality (2.1) for $C_{1}=\{x \in C:|x| \geqslant$ $1\}$. By rescaling, we may assume that $V<V_{0}$. For $i$ large, we may assume that $U_{i}$ is contained in $C_{1}$ and that

$$
m_{0} \geqslant \delta\left|\partial U_{i}\right|
$$

By (2.1), $\left|\partial U_{i}\right| \geqslant \delta \beta V^{n /(n+1)}$, and therefore

$$
m_{0} \geqslant \delta^{2} \beta V^{n /(n+1)} \text {. }
$$

On the other hand, an initial piece of $C$ of volume $V$ has perimeter

$$
\left(|M| /\left|\mathbb{S}^{n}\right|\right)^{1 /(n+1)} \beta V^{n /(n+1)}<\delta^{2} \beta V^{n /(n+1)},
$$

the desired contradiction.

The asserted regularity is standard ([M1, Thm. 8.6]).

Remark 2.3 (Hypercube and More Singular Cones). Gnepp, Ng, and Yoder GNY], CFG] have proved, for the surface of the cube, that for small prescribed area, a geodesic disc about a vertex minimizes the perimeter. The corresponding question is open for the hypercube in $\mathbb{R}^{4}$ or even for its tangent cone $C$ at a vertex, which has a 1-dimensional singular set consisting of four rays from the vertex.

Cao and Escobar CE study such three-dimensional PL Riemannian manifolds and prove isoperimetric inequalities, but their fundamental estimate, even if generalized from their case of nonpositive curvature to our case of nonnegative curvature, does not rule out, for example, a sphere which crosses each singular ray twice. 


\section{ISOPERIMETRIC DOMAINS IN CERTAIN CONES}

Let $C$ be the cone over a smooth compact submanifold $\left(M^{n}, d s^{2}\right)$ of the sphere $\mathbb{S}^{N} \subset \mathbb{R}^{N+1}$, and assume that the Ricci curvature is nonnegative.

The punctured cone $C^{*}=C-\{0\}$ can be viewed as a warped product $(0,+\infty) \times_{f}$ $M$, where $f(t)=t$, endowed with the Riemannian metric $\langle\rangle=,d t^{2}+t^{2} d s^{2}$. We consider on $C$ the radial vector field $X=t \partial / \partial t$. If $D$ is the Levi-Civitá connection on $C^{*}$, then $D_{u} X=u$ for any $u \in T C^{*}$. We refer to [ON, pp. 204-211] and [Mo] for background on these warped products. In particular, the Ricci curvature of the cone is given by

$$
\begin{aligned}
\operatorname{Ric}\left(\partial_{t}, \partial_{t}\right) & =0, \\
\operatorname{Ric}(u, u) & =\frac{1}{t^{2}}\left(\operatorname{Ric}_{M}(u, u)-(n-1)\right),
\end{aligned}
$$

for $u$ tangent to the geodesic sphere of radius $t>0$ centered at the vertex. Hence $\operatorname{Ric}_{M} \geqslant n-1$ is equivalent to Ric $\geqslant 0$.

We are interested in characterizing the isoperimetric domains in these cones. When $M$ is a curve, i.e. $C$ is a two-dimensional cone, then $C$ is isometric to a right circular cone, for which isoperimetric domains are the geodesic balls about the vertex. See [HHM, sect. 8] and Theorem 1.1

In arbitrary dimension $n \geqslant 2$, we know from Theorem [2.2 that $\partial U=\Sigma \cup$ $\Sigma_{0}$, where $\Sigma$ is a smooth hypersurface with inward constant mean curvature $H=$ $n^{-1}\left(k_{1}+\ldots+k_{n}\right)$ (average of principal curvatures) and $\mathcal{H}^{s}\left(\Sigma_{0}-\{0\}\right)=0$ for $s>n-7$. Moreover, at every point of $p \in \Sigma_{0}-\{0\}$ there is a tangent cone, and $|\sigma|^{2}=k_{1}^{2}+\ldots+k_{n}^{2}$ (the squared sum of the principal curvatures) goes to $\infty$ when we approach $p$ from inside $\Sigma$.

The regular part $\Sigma$ is a stable hypersurface for given volume. This means that the index form $Q$ satisfies the inequality

$$
Q(u, u)=-\int_{\Sigma} u\left(\Delta u+\left(\operatorname{Ric}(N, N)+|\sigma|^{2}\right) u\right) d \Sigma \geqslant 0,
$$

for any smooth function $u$ with mean zero and compact support in $\Sigma$ ([BdCE]). In the above formula, $\Delta$ is the Laplacian on $\Sigma$ and $N$ is the unit inner normal vector to $\Sigma$.

Lemma 3.1. Let $\Sigma^{k}(k \geqslant 2)$ be a smooth, embedded manifold of bounded mean curvature in $\mathbb{R}^{n+1}$ or in any cone $C$ over a smooth submanifold $M^{n}$ of the unit sphere $\mathbb{S}^{N}$, with singular set $\Sigma_{0}=\bar{\Sigma}-\Sigma$, satisfying $\mathcal{H}^{k-2}\left(\Sigma_{0}\right)=0$ or consisting of isolated points. Then, given $\varepsilon>0$, there is a smooth function $\varphi_{\varepsilon}: \bar{\Sigma} \rightarrow[0,1]$ supported in $\Sigma$ such that

(i) $\mathcal{H}^{k}\left(\left\{\varphi_{\varepsilon} \neq 1\right\}\right)<\varepsilon$,

(ii) $\int_{\Sigma}\left|\nabla \varphi_{\varepsilon}\right|^{2} d \Sigma<\varepsilon$

(iii) $\int_{\Sigma}\left|\Delta \varphi_{\varepsilon}\right| d \Sigma<\varepsilon$.

Remark 3.2. The idea of the proof is to use the definition of Hausdorff measure to obtain a covering of the singular set $\Sigma_{0}$ by small balls $B_{i}$, to choose functions $\varphi_{i}$ vanishing on $B_{i}$, and to set $\varphi_{\varepsilon}=\prod \varphi_{i}$. The problem is that the $B_{i}$ may overlap a lot. Leon Simon has explained to us that to obtain (ii), as in SSS, sect. 2], one could take $\varphi_{\varepsilon}=\min \varphi_{i}$. The reader can find a detailed argument in [SZ, Lemma 2.4]. To obtain both (ii) and (iii), we instead choose the $B_{i}$ carefully in order to bound overlap among balls of comparable size, and then divide the balls into size classes. 
Proof. We may assume that $\Sigma$ has compact closure $\bar{\Sigma}$. First we treat the case that $\mathcal{H}^{k-2}\left(\Sigma_{0}\right)=0$. Note that there is a constant $c_{1}(N) \geqslant 1$ such that, for any collection of balls $B\left(p_{i}, r_{i}\right)$ in $\mathbb{R}^{N+1}$ with radii within a factor of $4\left(\max r_{i} \leqslant 4 \min r_{i}\right)$ and the $B\left(p_{i}, r_{i} / 6\right)$ disjoint,

$$
\text { a ball intersects at most } c_{1} \text { balls, }
$$

as follows easily by a volume estimate.

Choose a smooth radial function $\varphi: \mathbb{R}^{N+1} \rightarrow[0,1]$ such that $\varphi$ vanishes on $B(0,1 / 2)$ and $\varphi=1$ on $B(0,9 / 10)^{C}$. Let $c_{2}=\sup \left\{|D \varphi|^{2},\left|D^{2} \varphi\right|\right\}$, where $D$ denotes differentiation in $\mathbb{R}^{N+1}$. Then scalings of $\varphi$ to any smaller $B(p, r)$, vanishing on $B(p, r / 2)$ and equal to 1 on $B(p, 9 r / 10)^{C}$, satisfy

$$
|D \varphi|^{2} \leqslant \frac{c_{2}}{r^{2}}, \quad\left|D^{2} \varphi\right| \leqslant \frac{c_{2}}{r^{2}} .
$$

We claim that on $\Sigma$, for some $c_{3} \geqslant c_{2}$, for a scaling to $\varphi_{0}$ on a small ball $B(0, r)$ about 0 ,

$$
\left|\Delta \varphi_{0}\right| \leqslant \frac{c_{3}}{r^{2}}
$$

Indeed, note that the radial mean curvature of $\Sigma$ in $\mathbb{R}^{N+1}$ equals its radial mean curvature in $C$, which is bounded by hypothesis by $H_{0}$, the bound on the mean curvature. Since $\varphi_{0}$ is radial,

$$
\left|\Delta \varphi_{0}\right| \leqslant N\left(\left|D^{2} \varphi_{0}\right|+H_{0}\left|D \varphi_{0}\right|\right) \leqslant N\left(\frac{c_{2}}{r^{2}}+H_{0} \frac{\sqrt{c_{2}}}{r}\right) \leqslant \frac{c_{3}}{r^{2}} .
$$

Since the radial mean curvature of $\Sigma$ in $\mathbb{R}^{N+1}$ is bounded, at the vertex one can apply monotonicity $\left(\left[\mathrm{S}\right.\right.$, Thm. 17.6], $\mathrm{A}$, Cor. 5.1(3)], [M1, 9.3]) in $\mathbb{R}^{N+1}$. Hence there is a constant $c_{4} \geqslant 1$ such that $\mathcal{H}^{k}(B(0, r) \cap \Sigma) \leqslant c_{4} r^{k}$ for $r \leqslant 1$.

Fix $\varepsilon>0$. Since $k \geqslant 3$, by (3.4a) we may scale down $\varphi_{0}$ to $B\left(0, r_{0}\right)$ with

$$
\mathcal{H}^{k}\left(B\left(0, r_{0}\right) \cap \Sigma\right)<\frac{\varepsilon}{2}, \quad \int_{B\left(0, r_{0}\right) \cap \Sigma}\left|\nabla \varphi_{0}\right|^{2}<\frac{\varepsilon}{16}, \quad \text { and } \int_{B\left(0, r_{0}\right) \cap \Sigma}\left|\Delta \varphi_{0}\right|<\frac{\varepsilon}{4} .
$$

On $B\left(0, r_{0} / 8\right)^{C}$ the curvature of $C$ is bounded, and the mean curvature of $\Sigma$ in $\mathbb{R}^{N+1}$ is bounded by say $H_{1}$. Hence by (3.4a), for some $c_{5}>c_{3}$, scalings of $\varphi$ to $B(p, r) \subset B\left(0, r_{0} / 8\right)^{C}$ satisfy

$$
|\Delta \varphi| \leqslant N\left(\left|D^{2} \varphi\right|+H_{1}|D \varphi|\right) \leqslant N\left(\frac{c_{2}}{r}+H_{1} \frac{\sqrt{c_{2}}}{r}\right) \leqslant \frac{c_{5}}{r^{2}}
$$

as does $\varphi_{0}$ (by $(3.4 \mathrm{~b})$ ). Also we may apply monotonicity to obtain $c_{6} \geqslant c_{4}$ such that for $|p| \geqslant r_{0} / 4$ and $r \leqslant r_{0} / 16$, as well as for $p=0$ and $r \leqslant 1$,

$$
\mathcal{H}^{k}(B(p, r) \cap \Sigma) \leqslant c_{6} r^{k}
$$

Cover $\Sigma_{0}-B\left(0, r_{0} / 2\right)$ with finitely many $B\left(p_{i}, r_{i} / 2\right)$ with $r_{0} / 16>r_{1} \geqslant r_{2} \geqslant \ldots$ such that

$$
\sum \alpha_{k} r_{i}^{k} \leqslant \frac{\varepsilon}{2} \quad \text { and } \quad \sum r_{i}^{k-2} \leqslant \frac{\varepsilon}{16 c_{1} c_{5} c_{6}}
$$

here $\alpha_{k}$ is the volume of the unit ball in $\mathbb{R}^{k}$. By covering first with $\left\{B\left(p_{i}, r_{i} / 6\right)\right\}$, enlarging, and discarding unnecessary balls, we may assume that the $B\left(p_{i}, r_{i} / 6\right)$ are disjoint. Divide this covering into classes $\mathcal{B}_{m}$ for which $2^{m} \leqslant r_{i}<2^{m+1}$. 
Put $\varphi_{\varepsilon}=\prod \varphi_{i}$. Conclusion (i) follows immediately by (3.5) and (3.7). We next show that

$$
\sum_{i \leqslant j} \int_{\Sigma}\left|\nabla \varphi_{i}\right|\left|\nabla \varphi_{j}\right|<\frac{\varepsilon}{4}
$$

Notice that by (3.4a) and (3.6) , for $i \leqslant j$,

$$
\int_{\Sigma}\left|\nabla \varphi_{i}\right|\left|\nabla \varphi_{j}\right| \leqslant \frac{c_{5}}{r_{i} r_{j}} c_{6} r_{j}^{k}
$$

We consider first the products involving $\nabla \varphi_{0}$. By (3.5), $\int_{\Sigma}\left|\nabla \varphi_{0}\right|^{2} \leqslant \varepsilon / 16$. By (3.8),

$$
\sum_{j \geqslant 1} \int_{\Sigma}\left|\nabla \varphi_{0}\right|\left|\nabla \varphi_{j}\right| \leqslant c_{5} c_{6} \sum_{j \geqslant 1} r_{j}^{k-2} \leqslant \frac{\varepsilon}{16},
$$

by (3.7). Therefore the products involving $\nabla \varphi_{0}$ contribute at most $\varepsilon / 8$ to (3.8).

Second, consider the remaining products involving $\nabla \varphi_{1}$. By (3.3) there are at most $c_{1}$ products with second factor from the largest class $\mathcal{B}_{m_{0}}$ and the next largest $\mathcal{B}_{m_{0}-1}$, yielding a contribution to (3.8) of at most $c_{1} c_{5} c_{6} r_{1}^{k-2}$ by (3.8]). Second factors from succeeding classes $\mathcal{B}_{m_{0}-1-h}$, with $r_{j} \leqslant 2^{-h} r_{1}$, contribute at most $c_{1} c_{5} c_{6} r_{1}^{k-2} \sum_{h} 2^{-h}=c_{1} c_{5} c_{6} r_{1}^{k-2}$, for a total of $2 c_{1} c_{5} c_{6} r_{1}^{k-2}$.

Similarly, the remaining products involving $\nabla \varphi_{2}$ contribute at most $2 c_{1} c_{5} c_{6} r_{2}^{k-2}$. Indeed, the further remaining products involving $\nabla \varphi_{i}$ contribute at most $2 c_{1} c_{5} c_{6} r_{i}^{k-2}$. Therefore

$$
\sum_{0 \leqslant i \leqslant j} \int_{\Sigma}\left|\nabla \varphi_{i}\right|\left|\nabla \varphi_{j}\right|<2 c_{1} c_{5} c_{6} \sum_{i \geqslant 1} r_{i}^{k-2}+\frac{\varepsilon}{8} \leqslant \frac{\varepsilon}{4}
$$

by (3.7), proving (3.8). Now

$$
\int_{\Sigma}\left|\nabla \varphi_{\varepsilon}\right|^{2} \leqslant 2 \sum_{i \leqslant j} \int_{\Sigma}\left|\nabla \varphi_{i}\right|\left|\nabla \varphi_{j}\right|<\frac{\varepsilon}{2}
$$

proving (ii).

Finally we estimate that

$$
\int_{\Sigma}\left|\Delta \varphi_{\varepsilon}\right| \leqslant \sum_{i} \int_{\Sigma}\left|\Delta \varphi_{i}\right|+2 \sum_{i \leqslant j} \int_{\Sigma}\left|\nabla \varphi_{i}\right|\left|\nabla \varphi_{j}\right|<\left(\sum_{i \geqslant 1} \frac{c_{5}}{r_{i}^{2}}\left(c_{6} r_{i}^{k}\right)+\frac{\varepsilon}{4}\right)+\frac{\varepsilon}{2}<\varepsilon,
$$

by (3.5), (3.6), (3.5), (3.8), and (3.7), and proving (iii).

Second, we consider the case when $\mathcal{H}^{k-2}\left(\Sigma_{0}\right)>0$ but $\Sigma_{0}$ consists of isolated points, so that $k=2$. It suffices to consider a small ball about a single point $p$ (trivially establishing (i) and guaranteeing that $\int H_{0}^{2}$ and $H_{0}$ times the diameter are small), which for convenience we move and scale to be $B(0,2)$. For this case we need a refined form of monotonicity involving the area

$$
A(r)=\mathcal{H}^{2}(B(0, r) \cap \Sigma)
$$

and the angle $\theta$ that the normal to $\Sigma$ makes with the radial direction:

$$
\frac{A\left(r_{2}\right)}{r_{2}^{2}} e^{H_{0} r_{2}}-\frac{A\left(r_{1}\right)}{r_{1}^{2}} e^{H_{0} r_{1}} \geqslant \int_{\Sigma \cap\left\{r_{1} \leqslant r \leqslant r_{2}\right\}} \frac{\cos ^{2} \theta}{r^{2}} d \Sigma
$$




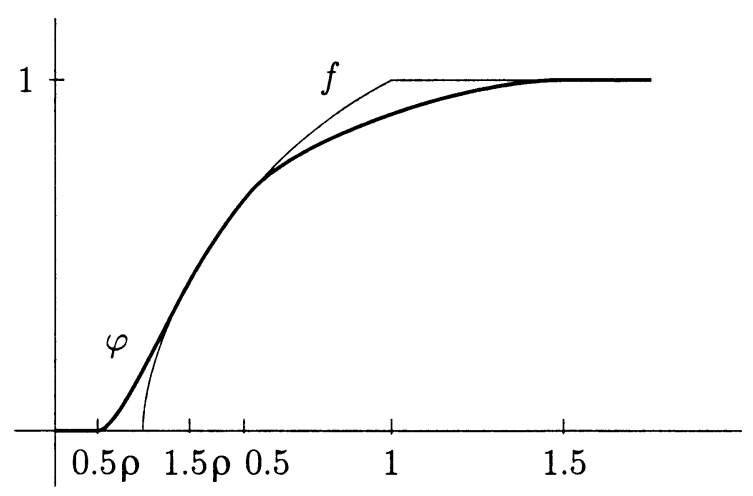

Figure 1. Dimension two requires a logarithmic cut-off function.

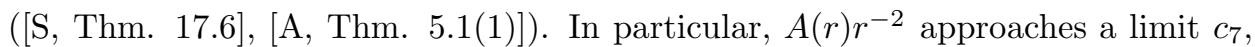
and we may assume it is close to that limit (by taking the original small ball small enough). Since $H_{0} r_{2}$ is small, it follows from (3.9) that

$$
\int_{B(0,2)} \frac{\cos ^{2} \theta}{r^{2}} d \Sigma
$$

is small.

For $\rho>0$ small, let

$$
f(r)= \begin{cases}0, & r \leqslant \rho, \\ \frac{\log (r / \rho)}{\log (1 / \rho)}, & \rho \leqslant r \leqslant 1, \\ 1, & 1 \leqslant r,\end{cases}
$$

as in Figure 1. Note that $f^{\prime}(r)=(r \log (1 / \rho))^{-1}$ and $f^{\prime \prime}(r)=-\left(r^{2} \log (1 / \rho)\right)^{-1}$ in the interval $[\rho, 1]$. We obtain a smooth function $\varphi$ by altering $f$ as in Figure 1 on $\left(\frac{1}{2} \rho, \frac{3}{2} \rho\right)$ and $\left(\frac{1}{2}, \frac{3}{2}\right)$. The function $\varphi$ is convex in the interval $\left(\frac{1}{2} \rho, \frac{3}{2} \rho\right)$ and concave in $\left(\frac{1}{2}, \frac{3}{2}\right)$, and hence $\varphi^{\prime}$ is positive in both intervals. We can even choose $\varphi$ so that there is an absolute constant $M>0$ such that $\varphi^{\prime \prime}$ is bounded above by $M\left(\rho^{2} \log (1 / \rho)\right)^{-1}$ in $\left(\frac{1}{2} \rho, \frac{3}{2} \rho\right)$, and bounded below by $-M(\log (1 / \rho))^{-1}$ in $\left(\frac{1}{2}, \frac{3}{2}\right)$.

As $\varphi^{\prime}$ is increasing in $\left(\frac{1}{2} \rho, \frac{3}{2} \rho\right)$ and decreasing in $\left(\frac{1}{2}, \frac{3}{2}\right)$, we have

$$
\varphi^{\prime} \leqslant 3(r \log (1 / \rho))^{-1} \text {. }
$$

Then, if $A(r)=\mathcal{H}^{2}(B(0, r) \cap \Sigma)$,

$$
\begin{aligned}
\log ^{2}(1 / \rho) \int_{\Sigma}|\nabla \varphi|^{2} & \leqslant 3 \int_{\rho / 2}^{3 / 2} \frac{d A}{r^{2}} \\
& =3\left[\frac{A(r)}{r^{2}}\right]_{\rho / 2}^{3 / 2}+3 \int_{\rho}^{3 / 2} \frac{2 A(r)}{r^{3}} d r \\
& \leqslant 9 c_{7}+12 c_{7} \log (3 / 2 \rho)
\end{aligned}
$$

because $A(r) \leqslant 2 c_{7} r^{2}$, so that $\int_{\Sigma}|\nabla \varphi|^{2} d \Sigma$ is small for $\rho$ small, proving (ii). 
To estimate $\int|\Delta \varphi|$, note that

$$
|\Delta \varphi| \leqslant\left|\varphi^{\prime \prime}\right|+H_{0}\left|\varphi^{\prime}\right| \leqslant\left|\varphi^{\prime \prime}\right|+.5\left|\varphi^{\prime}\right|^{2}+.5 H_{0}^{2} .
$$

Since $\int\left|\varphi^{\prime}\right|^{2}$ and $\int H_{0}^{2}$ are small, it suffices to estimate $\int\left|\varphi^{\prime \prime}\right|$. On the altered portion inside $\left(\frac{1}{2} \rho, \frac{3}{2} \rho\right)$,

$$
\int_{\Sigma \cap\left\{\frac{1}{2} \rho \leqslant r \leqslant \frac{3}{2} \rho\right\}} \varphi^{\prime \prime} \leqslant M \frac{1}{\rho^{2} \log (1 / \rho)} 2 c_{7}\left(\frac{3 \rho}{2}\right)^{2},
$$

which is small for $\rho$ small. Similarly, on the altered portion inside $\left(\frac{1}{2}, \frac{3}{2}\right)$,

$$
\int_{\Sigma \cap\left\{\frac{1}{2} \leqslant r \leqslant \frac{3}{2}\right\}} \varphi^{\prime \prime} \leqslant M \frac{1}{\log (1 / \rho)} 2 c_{7}\left(\frac{3}{2}\right)^{2},
$$

which is small for $\rho$ small.

On the unaltered portion, where $\varphi=f, \Delta \varphi=\Delta f$. In $\mathbb{R}^{N+1}$, at say $(r, 0,0, \ldots)$, $f_{11}=-\left(r^{2} \log (1 / \rho)\right)^{-1}, f_{22}=\ldots=\left(r^{2} \log (1 / \rho)\right)^{-1}$. Hence in $\Sigma$,

$$
\Delta \varphi \leqslant \frac{2 \cos ^{2} \theta}{r^{2}}+2 H_{0}|\nabla f| \leqslant \frac{2 \cos ^{2} \theta}{r^{2}}+|\nabla f|^{2}+H_{0}^{2},
$$

which has a small integral by (3.10), (3.11), and the smallness of the integral of $H_{0}^{2}$, proving (iii).

Lemma 3.3. Let $\Sigma^{n}(n \geqslant 2)$ be a smooth, bounded hypersurface of constant mean curvature in the cone $C$ over a smooth submanifold $M^{n}$ of the sphere $\mathbb{S}^{N}$, with singular set $\Sigma_{0}=\bar{\Sigma}-\Sigma$ satisfying $\mathcal{H}^{n-2}\left(\Sigma_{0}\right)=0$ or consisting of isolated points.

If $\Sigma$ is stable, then inequality (3.2) holds for any smooth bounded function $u$ : $\Sigma \rightarrow \mathbb{R}$ with mean zero on $\Sigma$ and gradient in $L^{2}(\Sigma)$. Moreover, $\int_{\Sigma}|\sigma|^{2} d \Sigma$ is finite.

Proof. If $u$ is a bounded function with mean zero on $\Sigma$ and $L^{2}$ gradient on $\Sigma$, then define $u_{\varepsilon}=\left(\varphi_{\varepsilon} u\right)^{+}-a_{\varepsilon}\left(\varphi_{\varepsilon} u\right)^{-}$, where $a_{\varepsilon}$ is a constant computed so that $u_{\varepsilon}$ has mean zero over $\Sigma$. As $u$ has mean zero, it follows that $a_{\varepsilon} \rightarrow 1$ when $\varepsilon \rightarrow 0$. Then inequality (3.2) holds for $u_{\varepsilon}$, since $u_{\varepsilon}$ has compact support on $\Sigma$. As $\varepsilon \rightarrow 0$, it follows by Lemma 3.1 that (3.2) holds for $u$ as well.

To show the finiteness of the integral $\int_{\Sigma}|\sigma|^{2} d \Sigma$, consider a function $u \equiv 1$ in a neighborhood $V$ of $\Sigma_{0}$ in $\Sigma$ and extend it so that $|u| \leqslant 1,|\nabla u|$ is bounded and $\int_{\Sigma} u d \Sigma=0$. Then, by the first part of this lemma and (3.2),

$$
\int_{V}|\sigma|^{2} d \Sigma \leqslant \int_{\Sigma}|\nabla u|^{2} d \Sigma<\infty
$$

We now prove Minkowski formulae for the regular part, $\Sigma$. The reader can consult Montiel's paper ([Mo, §5]) for the smooth case.

Proposition 3.4 (Minkowski formulae on $\Sigma)$. Let $\Sigma^{n}(n \geqslant 2)$ be a smooth, bounded hypersurface of constant mean curvature in the cone $C$ over a smooth submanifold $M^{n}$ of the sphere $\mathbb{S}^{N}$, with singular set $\Sigma_{0}=\bar{\Sigma}-\Sigma$ satisfying $\mathcal{H}^{n-2}\left(\Sigma_{0}\right)=0$ or $\Sigma_{0}=\{0\}$. Then

$$
\int_{\Sigma}\{1+H\langle X, N\rangle\} d \Sigma=0,
$$


where $N$ is the inner normal to $\Sigma$. If, in addition, $H$ is constant, then

$$
\int_{\Sigma}\left\{\operatorname{Ric}(N, N)+\left(|\sigma|^{2}-n H^{2}\right)\right\}\langle X, N\rangle d \Sigma=0 .
$$

Formula (3.12) is the first Minkowski formula, and (3.13) is the second Minkowski formula.

Proof. Let $X^{T}$ be the tangent projection of the conformal field $X$ to $\Sigma$. Consider the functions $\varphi_{\varepsilon}$ defined in Lemma 3.1, which have compact support in $\Sigma$. By the divergence theorem the integral of the vector field $\operatorname{div}_{\Sigma}\left(\varphi_{\varepsilon} X^{T}\right)$ over $\Sigma$ is 0 . Letting $\varepsilon \rightarrow 0$ and using Lemma 3.1 we obtain that $\operatorname{div}_{\Sigma}\left(X^{T}\right)=n(1+H\langle X, N\rangle)$ has mean zero over $\Sigma$, which proves (3.12).

Now we consider the variation of $\Sigma$ with initial velocity vector field $\varphi_{\varepsilon} N$, where $N$ is the inner normal to $\Sigma$. Let $\Sigma_{t}$ be the hypersurface obtained at time $t$. Over $\Sigma_{t}$ the first Minkowski formula holds, so that, differentiating with respect to $t$ and evaluating at $t=0$, we have

$$
0=\left.\int_{\Sigma}(1+H\langle X, N\rangle) \frac{d}{d t}\right|_{t=0} d \Sigma_{t}+\int_{\Sigma}\left\{\left.\langle X, N\rangle \frac{d}{d t}\right|_{t=0} H_{t}+\left.H \frac{d}{d t}\right|_{t=0}\langle X, N\rangle\right\} d \Sigma
$$

and we have

$$
\begin{aligned}
& \left.\frac{d}{d t}\right|_{t=0} d \Sigma_{t}=-n H\left\langle\varphi_{\varepsilon} N, N\right\rangle=-n H \varphi_{\varepsilon}, \\
& \left.\frac{d}{d t}\right|_{t=0} H_{t}=\frac{1}{n}\left(\Delta \varphi_{\varepsilon}+\left(\operatorname{Ric}(N, N)+|\sigma|^{2}\right) \varphi_{\varepsilon}\right), \\
& \left.\frac{d}{d t}\right|_{t=0}\langle X, N\rangle=\varphi_{\varepsilon} .
\end{aligned}
$$

The first equality is standard when computing the first derivative of the perimeter. The second one is a well known formula for the derivative of the mean curvature along a deformation. The third one is immediate. Substituting in (3.14), we obtain

$$
\begin{aligned}
0=\int_{\Sigma} \frac{1}{n}\left(\Delta \varphi_{\varepsilon}+(\operatorname{Ric}(N, N)\right. & \left.\left.+|\sigma|^{2}\right) \varphi_{\varepsilon}\right)\langle X, N\rangle \\
& +\int_{\Sigma} H \varphi_{\varepsilon}-\int_{\Sigma} n H \varphi_{\varepsilon}(1+H\langle X, N\rangle) .
\end{aligned}
$$

Letting $\varepsilon \rightarrow 0$, using Lemma 3.1 and the first Minkowski formula (3.12), we obtain (3.13).

Remark 3.5. The function $u=1+H\langle X, N\rangle$ is bounded over (the bounded) $\Sigma$, and its gradient equals

$$
\sum_{i} H\left\langle X, k_{i} e_{i}\right\rangle e_{i}
$$

where $e_{i}\left(\left|e_{i}\right|=1\right)$ are principal directions with principal curvatures $k_{i}$. So the modulus of the gradient of $u$ is bounded from above by $H|X||\sigma|$, which is in $L^{2}(\Sigma)$. If $\Sigma$ is stable, then $Q(u, u) \geqslant 0$ by Lemma 3.3

Theorem 3.6. Let $\Sigma^{n}(n \geqslant 2)$ be a smooth, bounded, stable hypersurface of constant mean curvature in the cone $C$ over a smooth, connected submanifold $M^{n}$ of the sphere $\mathbb{S}^{N}$, with Ricci curvature Ric $\geqslant 0$. Suppose that the singular set $\Sigma_{0}=\bar{\Sigma}-\Sigma$ 
satisfies $\mathcal{H}^{n-2}\left(\Sigma_{0}\right)=0$ or $\Sigma_{0}=\{0\}$. Then either $\Sigma$ is a geodesic sphere centered at the vertex of the cone, or $\Sigma$ bounds a flat round ball.

Theorem [3.6 can fail for $n=1$, because if the vertex angle is a multiple of $2 \pi$, you can have a constant-curvature curve encircling the vertex not centered at the vertex.

Proof. The function $u=1+H\langle X, N\rangle$, where $N$ is the inward normal to $\Sigma$, has mean zero over $\Sigma$ by the first Minkowski formula (3.12). Observe that $H \neq 0$.

We first note that $\Sigma$ is connected. This can be proved by inserting a locally constant nowhere vanishing function $v$ over $\Sigma$ in the index form $Q$. By Lemma 3.3 we have $Q(v, v) \geqslant 0$, and so $\operatorname{Ric}(N, N)+|\sigma|^{2} \equiv 0$, which implies $H=0$, a contradiction to the first paragraph.

A straightforward calculation as in $[\mathrm{BdC}$, Lemmas 3.5 and 2.23] (the only modification is that the Ricci curvature appears in formula (i) of Lemma 2.23) shows that

$$
\Delta u+\left(\operatorname{Ric}(N, N)+|\sigma|^{2}\right) u=\left(\operatorname{Ric}(N, N)+\left(|\sigma|^{2}-n H^{2}\right)\right) .
$$

By Remark 3.5 inequality (3.2) holds for $u$, and we have

$$
-\int_{\Sigma}\left\{\operatorname{Ric}(N, N)+\left(|\sigma|^{2}-n H^{2}\right)\right\}(1+H\langle X, N\rangle) d \Sigma \geqslant 0 .
$$

From the second Minkowski formula (3.13) we deduce

$$
-\int_{\Sigma}\left\{\operatorname{Ric}(N, N)+\left(|\sigma|^{2}-n H^{2}\right)\right\} d \Sigma \geqslant 0
$$

As $|\sigma|^{2}-n H^{2} \geqslant 0$ and $\operatorname{Ric}(N, N) \geqslant 0$, we obtain that $\operatorname{Ric}(N, N)=0$ and $|\sigma|^{2}=$ $n H^{2}$, so that $\Sigma$ is totally umbilic. Furthermore, $\Sigma_{0}$ is empty since $|\sigma|^{2}$ is bounded. We conclude by applying Lemma 3.8

Remark 3.7. Instead of the Minkowski formulas, one could just use scaling and unit normal variations.

Earlier work by Montiel [Mo, Cor. 7] considered more general, say non-constantcurvature, warped products and showed that a smooth, compact, constant-meancurvature hypersurface which is a graph over one of the constant-mean-curvature slices must be such a slice. For cones, the graph hypothesis is unnecessary (see our Lemma 3.81) and his additional hypothesis on the Ricci curvature reduces to ours. Our Lemma 3.3 shows that Montiel's results extend to hypersurfaces with mild singularities.

Lemma 3.8. Let $\Sigma^{n}(n \geqslant 2)$ be a smooth, compact, connected, totally umbilic hypersurface of constant nonzero mean curvature in the cone $C$ over a smooth, connected submanifold $M^{n}$ of the sphere $\mathbb{S}^{N}$. Then either $\Sigma$ is a geodesic sphere centered at the vertex of the cone, or $\Sigma$ bounds a flat round ball.

Proof. We reproduce Montiel's arguments [Mo, pp. 732-733] since the result is not explicitly stated in his paper. Let $N$ be the inward normal to $\Sigma$. As $\Sigma$ is totally umbilic, we have $\nabla_{\Sigma}^{2}\langle X, N\rangle=-\left(H^{2}\langle X, N\rangle+H\right)\langle$,$\rangle . So \langle X, N\rangle$ is a concircular scalar field on $\Sigma$ in the sense of Tashiro's paper [T].

If the function $H^{2}\langle X, N\rangle+H$ is identically 0 over $\Sigma$, then $\Sigma$ is a geodesic sphere about the vertex, since $M$ is connected $(\langle X, N\rangle$ is the same at maxima and minima 
of distance to the vertex; hence that distance must be constant). Otherwise, by $\mathrm{T}$. Thm. 2 (III)], $\Sigma$ is a sphere with sectional curvature $H^{2}$, because $\Sigma$ is connected.

Let $R, R_{\Sigma}$ denote the curvature operators in $C$ and $\Sigma$, respectively. We are going to show that $R \equiv 0$ over $\Sigma$. First observe that $R(u, v) X=0$ for any $u, v \in T C^{*}$, and $R(u, v) w=0$ for all $u, v, w \in T \Sigma$. The last equality follows from the Gauss equation taking into account that $\Sigma$ is totally umbilic and that $R_{\Sigma}$ is the curvature operator of a sphere with sectional curvature $H^{2}$. This implies that $R(u, v) w=0$ for any $u, v, w \in T C^{*}$ along $\{x \in \Sigma:\langle X, N\rangle \neq 0\}$ (when $X$ is not tangent to $\Sigma$ ). But when $X$ is tangent to $\Sigma(\langle X, N\rangle=0)$ we have $\left\langle\nabla_{\Sigma}\langle X, N\rangle, X\right\rangle=-H|X|^{2}$, which is different from 0 out of the vertex; so $\{p \in \Sigma:\langle X, N\rangle=0\}$ is a hypersurface of $\Sigma$ and $R$ vanishes identically on $\Sigma$.

Projecting $\Sigma$ radially to $M$, we obtain a set $\Omega$. Taking into account the relation between curvatures in $C$ and in $M$, we conclude that the sectional curvatures of $M$ over $\Omega$ equal 1 , and so $\operatorname{Ric}_{M}=n-1$ on $\Omega$.

If $\Sigma$ encloses the vertex of the cone, then $\Omega$ is all of $M$ and the cone is Euclidean (without a singularity). Otherwise $(0, \infty) \times \Omega$ is a region with zero sectional curvature containing $\Omega$ and the region it encloses. One can contract $\Sigma$ by its inner normal to conclude that $\Sigma$ bounds a flat cone with no singularity, i.e., a Euclidean region, which must be a round, flat ball.

Corollary 3.9 (Isoperimetric Theorem). Let $C$ be a cone with nonnegative Ricci curvature over a connected submanifold $M^{n}$ of the sphere $\mathbb{S}^{N}(n \geqslant 2)$. Then geodesic spheres about the vertex uniquely minimize perimeter for given volume (unless $\left.C=\mathbb{R}^{n+1}\right)$.

Proof. As Ric $\geqslant 0$, we have $\operatorname{Ric}_{M} \geqslant n-1$ by (3.1). Since $n \geqslant 2$, by Bishop's Theorem [C, Theorem. 3.9], $\left|M^{n}\right| \leqslant\left|\mathbb{S}^{n}\right|$, and equality implies that $M$ is isometric to $\mathbb{S}^{n}$ and so $C=\mathbb{R}^{n+1}$. Hence we may assume that $\left|M^{n}\right|<\left|\mathbb{S}^{n}\right|$. Combine Theorems 2.2 and 3.6 to obtain an isoperimetric domain which is either a geodesic ball about the vertex or a ball with zero sectional curvature enclosed by a totally umbilic hypersurface. A domain of the latter type satisfies the Euclidean isoperimetric inequality. Direct comparison using $\left|M^{n}\right|<\left|\mathbb{S}^{n}\right|$ shows that a geodesic sphere about the vertex of the same volume has less perimeter.

Remark 3.10. Corollary [3.9] is sharp in the sense that if $M$ is a round sphere, then geodesic spheres have nonnegative second variation for fixed volume if and only if Ric $\geqslant 0$, and if Ric $=0$ (so that $C$ is $\mathbb{R}^{n}$ ), then isoperimetric domains are not unique.

It is still an open question, however, if the Ricci curvature hypothesis could be weakened to the hypothesis $|M|<\left|\mathbb{S}^{n}\right|$ of the Existence Theorem 2.2.

Remark 3.11. Bray and Morgan BrM apply Corollary 3.9 to identify isoperimetric domains in certain warped products $I \times M$, such as Schwarzschild space.

Corollary 3.12. Let $C$ be a cone with nonnegative Ricci curvature over a connected submanifold $M^{n}$ of the sphere $\mathbb{S}^{N}(n \geqslant 2)$. Then horizontal slices of an isoperimetric region in $C \times \mathbb{R}^{m}$ are geodesic spheres.

Proof. Otherwise symmetrization, replacing horizontal slices with geodesic spheres, would decrease perimeter while preserving volumes. 
Remark 3.13. Similarly if $n=1$ and $|M|<2 \pi$, then of course geodesic circles about the origin uniquely minimize perimeter in $C$, and isoperimetric regions in $C \times \mathbb{R}^{m}$ are geodesic spheres about points in $\{0\} \times \mathbb{R}^{m}$.

Remark 3.14. Morgan [M2] shows that $n$-dimensional area-minimizing hypersurfaces in cones sometimes pass through the vertex if $n \geqslant 3$.

\section{REFERENCES}

[A] W. K. Allard, On the first variation of a varifold, Ann. of Math., 95 (1972) 417-491. MR 46:6136

[BdC] J. L. Barbosa and M. do Carmo, Stability of hypersurfaces with constant mean curvature, Math. Z. 185 (1984) 339-353. MR 85k:58021c

[BdCE] J. L. Barbosa, M. do Carmo and J. Eschenburg, Stability of hypersurfaces with constant mean curvature in Riemannian manifolds, Math. Z., 197 (1988) 123-138. MR 88m:53109

[BM] P. Bérard, D. Meyer, Inégalités isopérimétriques et applications, Ann. Scient. Éc. Norm. Sup. (4), 15 (1982) 513-542. MR 84h:58147

[Br] H. Bray, The Penrose inequality in general relativity and volume comparison theorems involving scalar curvature, Ph. D. Thesis, Stanford University, 1997.

[BrM] H. Bray, F. Morgan, An isoperimetric comparison theorem for Schwarzchild space and other manifolds, Proc. Amer. Math. Soc., to appear.

[CE] J. Cao and J. F. Escobar, A new 3-dimensional curvature integral formula for PLmanifolds of nonpositive curvature, preprint, 2000.

[C] I. Chavel, Riemannian geometry: a modern introduction, Cambridge Tracts in Mathematics, no. 108, Cambridge University Press, 1993. MR 95j:53001

[CFG] A. Cotton, D. Freeman, A. Gnepp, T. Ng, J. Spivack, C. Yoder (Williams College NSF "SMALL" undergraduate research Geometry Groups 1998, 2000), The isoperimetric problem on singular surfaces, preprint (2000).

[F] H. Federer, Geometric measure theory, Grundlehren Math. Wissen. 153, Springer-Verlag, New York, 1969. MR 41:1976

[GNY] A. Gnepp, T. F. Ng, C. Yoder, Isoperimetric domains on polyhedra and singular surfaces, NSF "SMALL" undergraduate research Geometry Group report, Williams College, 1998.

[HHM] H. Howards, M. Hutchings, F. Morgan, The isoperimetric problem on surfaces, Amer. Math. Monthly, 106, no. 5, (1999) 430-439. MR 2000i:52027

[Mo] S. Montiel, Unicity of constant mean curvature hypersurfaces in foliated Riemannian manifolds, Indiana Univ. Math. J., 48, no. 2, (1999) 711-748. MR 2001f:53131

[M1] F. Morgan, Geometric measure theory: a beginner's guide. Third edition, Academic Press, 2000. MR 2001j:49001

[M2] F. Morgan, Area-minimizing surfaces in cones, Comm. Anal. Geom., to appear.

$[\mathrm{MJ}]$ F. Morgan, D. Johnson, Some sharp isoperimetric theorems for Riemannian manifolds, Indiana Univ. Math. J., 49 (2000) 1017-1041.

[ON] B. O'Neill, Semi-Riemmanian geometry, Academic Press, New York, 1983. MR 85f:53002

[P] R. Pedrosa, The isoperimetric problem in spherical cylinders, preprint, 2002.

[PR] R. Pedrosa, M. Ritoré, Isoperimetric domains in the Riemannian product of a circle with a simply connected space form and applications to free boundary problems, Indiana Univ. Math. J., 48 (1999) 1357-1394. MR 2001k:53120

[R1] M. Ritoré, Applications of compactness results for harmonic maps to stable constant mean curvature surfaces, Math. Z., 226 (1997) 465-481. MR 98m:53082

[RR] M. Ritoré, A. Ros, Stable constant mean curvature tori and the isoperimetric problem in three space forms, Comment. Math. Helv., 67 (1992) 293-305. MR 93a:53055

[SS] R. Schoen, L. Simon, Regularity of stable minimal hypersurfaces, Comm. Pure App. Math., 34 (1981) 741-797. MR 82k:49054

[S] L. Simon, Lectures on geometric measure theory, Proc. Centre Math. Anal. 3, Australian National University, 1983. MR 87a:49001 
[SZ] P. Sternberg, K. Zumbrun, On the connectivity of boundaries of sets minimizing perimeter subject to a volume constraint, Comm. Anal. Geom., 7, no. 1, (1999) 199-220. MR 2000d:49062

[T] Y. Tashiro, Complete Riemannian manifolds and some vector fields, Trans. Amer. Math. Soc., 117 (1965) 251-275. MR 30:4229

Department of Mathematics, Williams College, Williamstown, Massachusetts 01267

E-mail address: Frank.Morgan@williams.edu

Departamento de Geometría y Topología, Universidad de Granada, E-18071 Granada, ESPAÑA

E-mail address: ritore@ugr.es 